\title{
Information Foraging in Nuclear Power Plant Control Rooms
}

\section{ESREL 2011}

\author{
R. L. Boring
}

\section{September 2011}

The INL is a

U.S. Department of Energy

National Laboratory

operated by

Battelle Energy Alliance

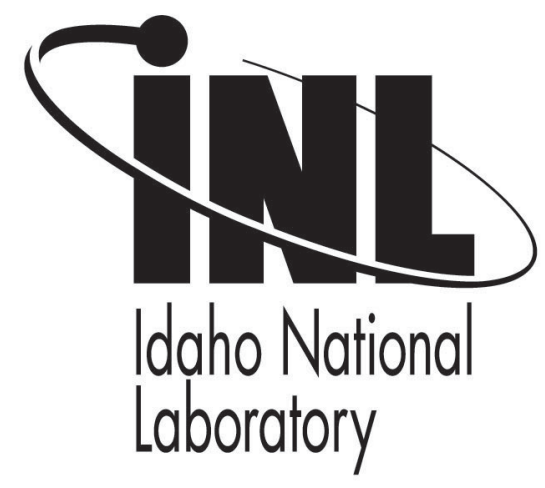

This is a preprint of a paper intended for publication in a journal or proceedings. Since changes may be made before publication, this preprint should not be cited or reproduced without permission of the author. This document was prepared as an account of work sponsored by an agency of the United States Government. Neither the United States Government nor any agency thereof, or any of their employees, makes any warranty, expressed or implied, or assumes any legal liability or responsibility for any third party's use, or the results of such use, of any information, apparatus, product or process disclosed in this report, or represents that its use by such third party would not infringe privately owned rights. The views expressed in this paper are not necessarily those of the United States Government or the sponsoring agency. 


\title{
Information foraging in nuclear power plant control rooms
}

\author{
R.L. Boring \\ Idaho National Laboratory, Idaho Falls, ID, US
}

\begin{abstract}
Information foraging theory articulates the role of the human as an "informavore" that seeks information and follows optimal foraging strategies (i.e., the "information scent") to find meaningful information. This paper briefly reviews the findings from information foraging theory outside the nuclear domain and then discusses the types of information foraging strategies operators employ for normal and off-normal operations in the control room. For example, operators may employ a predatory "wolf" strategy of hunting for information in the face of a plant upset. However, during routine operations, the operators may employ a trapping "spider" strategy of waiting for relevant indicators to appear. This delineation corresponds to information pull and push strategies, respectively. No studies have been conducted to determine explicitly the characteristics of a control room interface that is optimized for both push and pull information foraging strategies, nor has there been empirical work to validate operator performance when transitioning between push and pull strategies. This paper explores examples of control room operators as wolves vs. spiders and concludes by proposing a set of research questions to investigate information foraging in control room settings.
\end{abstract}

\section{INTRODUCTION}

Information foraging theory (Pirolli, 2007) articulates the role of the human as an "informavore" that seeks information and follows optimal foraging strategies (i.e,, the "information scent") in finding meaningful information. This theory has been successfully applied to human-information interaction environments such as Internet use, in which the informavorous Web user follows the information scent that yields the highest payoff. For example, when searching for particular information in the Internet, the Web user may go back and forth between the search engine and the identified sites in a pattern that closely mimics foraging for food between patches of optimally fruitful resources.

There are considerable differences between consumer Internet surfing and operator interactions with control rooms in nuclear power plants. $A$ major difference is that the information in control rooms has already been distilled to only the information that is relevant to some aspect of operations. Nonetheless, information needs vary considerably across different power and operation modes of the plant, and the operator needs to navigate to the most relevant information amid an abundance of plant indicators. Especially in emergency operations, the ability to find the relevant information on plant states requires specific information search strategies. Overreliance on single indicators is an example in which information search strategies have been optimized in a manner that may prove suboptimal in particular plant situations.

Digital control rooms present the opportunity to move away from the static data placement of analog control rooms, but such designs have not explicitly been optimized to account for the different information search strategies the operators may experience. In fact, if information becomes nested in windowing displays, the need to search for information may actually be increased, introducing a new set of information foraging strategies into the control room.

This paper briefly reviews the findings from information foraging theory outside the nuclear domain and then discusses the types of information foraging strategies operators employ for normal and off-normal operations in the control room. For example, operators may employ a predatory "wolf" strategy of hunting for information in the face of a plant upset. However, during routine operations, the operators may employ a trapping "spider" strategy of waiting for relevant indicators to appear. This delineation corresponds to information pull and push strategies, respectively, both of which are found in the control room. Yet, no studies have been conducted to determine explicitly the characteristics of a control room interface that is optimized for both push and pull information foraging strategies, nor has there been empirical work to validate operator performance when transitioning between push and pull strategies. 
This paper explores four examples of control room operators as wolves vs. spiders in terms of information foraging:

- Cases of information masking, in which the plant provides specific indicators of plant status, but these indicators may be absent or misleading. Such incidents are examples of operators following the wrong information scent or overrelying on a particular patch of informationi.e., over-foraging.

- Display layouts that optimize for foraging strategies in operator searches for information. Failing to provide indicators along a relevant foraging path may result in operators consistently overlooking or ignoring these indicators. While in practice, this is not different than designing a good layout, information foraging offers a sound theoretical basis for explaining good display layout as one optimized for information search strategies.

- Automation of plant functions, in which operator engagement is lost with some automation systems. By applying a varying process of push and pull information display, it is possible to help maintain operator engagement through creating a dynamic interaction between the plant and the operator.

- Alarm response, in which current annunciator systems feature a high number of nuisance alarms, which drive operators down the wrong information path. Similarly, alarm flooding results in an overabundance of push information. The problem may be recast not simply as information overload but as information scent overload. The key to effective alarm systems may be the effective management of the information scent provided to the operator.

Information foraging strategies are reviewed in terms of how they increase or decrease the operators' opportunity for successful operations. This paper concludes by proposing a set of research questions to investigate information foraging in control room settings.

\section{INFORMATION FORAGING}

\subsection{Foraging in animals and humans}

Foraging entails the search for food, and foraging theory is the subfield of behavioral ecology focused on how animals and humans optimally look for food (Marlow, 2005). Classic foraging theory predicts that the search for food in organisms is guided by the highest fitness payoff, consisting of the highest food yield coupled with the lowest cost of acquiring food. Foraging theory suggests that foraging strategies are adapted to maximize the fitness payoff and that it is possible to predict foraging strategies.

Optimal foraging theory (Stephens \& Krebs, 1986) classifies foraging behaviors according to the type of forager:

- Predators/Hunters: Attack and kill large numbers of food sources (i.e., their prey) and eat most or all of the food source.

- Grazers/Gatherers: Attack large numbers of food sources but only eat part of the food source.

- Parasitoids: Attack a single food source and eat most or all of the food source. The organisms may incubate in the food source and depart from the food source once its food content is depleted. The parasitoid may metamorphose into another form to facilitate the departure from the food source.

- Parasites: Attack a single food source but only eat part of the food source. They reside with or at that food source.

According to optimal foraging theory, the organism attempts to maximize the energy (E) received from a food source in proportion to the handling (h) and search (s) time:

$\frac{E}{(b+s)}$

Generalist organisms tend to have short search times, because they can ingest a variety of food sources. Specialist organisms tend to take longer to search, because they must find a particular food source. Foraging is affected by factors such as the density of food sources, which affects the overall search time. Within Optimal Foraging Theory, there are several types of foraging strategies discussed in the research literature, including (Kamil et al., 1987):

- Optimal Diet Model: The forager encounters different types of food sources and must choose which to pursue.

- Patch Selection Theory: The food source is concentrated in small areas with significant travel time between them, forcing the forager to select between food patches.

- Central Place Foraging Theory: The forager must return to a particular place in order to consume its food or perhaps to hoard it or feed it to a mate or offspring.

\subsection{Value beyond food}

In information foraging theory, the jumping off point from conventional foraging theory is 
the assumption that not everything of value is food. Behaviorism within psychology was early to acknowledge that there were different types of rewards. The operant conditioning paradigm talked of primary vs. secondary reinforcers (Skinner, 1974). A primary reinforcer, such as food, sleep, and sex, innately triggers a positive response that strengthens behaviors. A secondary reinforcer is a learned trigger of a positive response, one that must be paired with a primary reinforcer. For example, money is not in and of itself rewarding to humans, but humans learn to associate money with rewarding outcomes. The humanistic tradition in psychology similarly identified items of value to humans beyond food. In Maslow's Hierarchy of Needs (1943), basic physiological needs such as food are primary to the survival of the individual, but additional needs and desires such as safety, love and group belonging, and esteem are enabled when basic needs are met. Finally, within decision making theory, expected utility theory proposes that human decisions are governed by weighting the utility or value of expected outcomes (Von Neumann \& Morgenstern, 1947). Expected utility theory accounts for risky decisions such as taking chances, in which the chance of success is low but the outcome, if achieved, is significantly positive.

While neither behaviorism, humanistic psychology, nor expected utility theory is without critios these psychological frameworks serve to establish that human cognition and behavior are not governed solely by the need for food. With this backdrop, Pirolli (2007) proposes that information is of value to humans. In fact, just as the carnivore hunts for prey, Pirolli suggests that humans seek and value information. Pirolli coined the term informavore to explain the relation of the human to information. Just as humans develop different foraging strategies in the wild for food sources, humans develop different information seeking strategies depending on the need for and quality of information sources.

Information foraging theory encompasses any activities associated with searching for and handling information sources. Equation 1 from conventional foraging theory can be adapted as follows:

$$
\frac{I}{(h+s)}
$$

where I represents the information received from the source. Information utility may be seen as the product of the signal-to-noise ratio: a productive information search is one that yields high information content given the search time, whereas an unproductive information search is one that yields low information content given the search time.

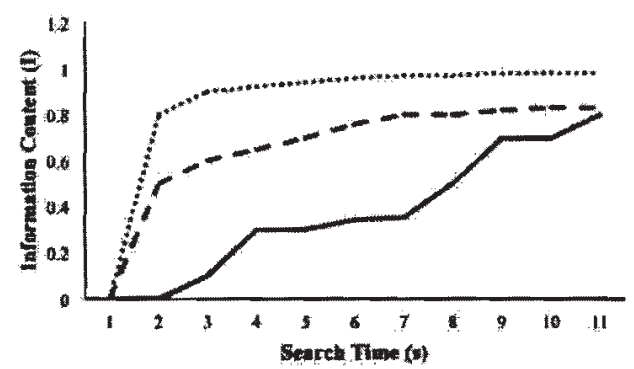

Figure 1. Different information foraging outcomes for Information Content (I) as a product of Search Time (s).

Figure 1 depicts different types of information yields as a product of search time. According to information foraging selection strategies, individuals would be more likely to select the high yield search target (as depicted by the dotted line) vs. the lower yield search targets (as depicted by the dashed and solid lines). In general terms, the probability of finding the right information increases as more search time is expended. However, note that all information reaches a point of diminishing returns at which time additional search will not unveil additional information. The likelihood of staying on a particular search path varies as a function of the quality of the information scent, and humans will adopt a new information search strategy if the information yield is not fruitful.

\section{INFORMATION FORAGING IN CONTROL ROOMS}

\subsection{Information wolves and spiders}

In the natural world, there exist two archetypical predators - the wolf and the spider. The wolf is a hunter who actively looks for its prey. In contrast, the spider builds a nest and waits for its prey to come to it. By analogy, an information wolf is someone who actively seeks information and may be said to pull information from available sources. An information spider is someone who receives (and in many cases subscribes to) information that is relevant. In the arachnoid case, it is said that information is pushed to the individual for appropriate use.

In informavores, being an information wolf or spider is not mutually exclusive, and humans may alternate between pulling information and receiving pushed information. The same can be said of control room operators in nuclear power plants. The temptation may be to oversimplify the role of the operator as one who either receives control room 
indications, signals, and alarms (i.e., the operator as spider) or actively monitors them (i.e., the operator as wolf). In fact, both activities are required for the successful operation of the plant. The control room operator must take in information from the control room instrumentation but also actively anticipate and diagnose changes in plant states.

The process of managing information in the control room is not static. As depicted in Figure 2, a typical nuclear power plant control room features push information sources like alarms as well as indicators, gauges, and displays from which the operator must actively pull information. For example, the operator as spider may receive an alarm that tells him or her to look for specific information. As the operator looks for confirmatory or additional information signaled by the alarm, the operator becomes an information wolf. The operator as wolf may change the type of information he or she is seeking, changing information foraging patterns in the control room to reflect different plant states. In this context, the changing plant states provide an information scent to look for additional information. Procedures may guide the operator to change from spider to wolf or vice versa as the situation dictates.

\subsection{Examples of information foraging in control rooms}

In the following sections, I explore four potential applications of information foraging in control rooms. The purpose is to review the applicability and utility of using a model adapted from animal behavior to explain operator performance in control rooms.

\subsubsection{Information masking}

Information masking occurs when the plant provides specific indicators of plant status, but the most relevant indicators may be missing or misleading. For example, in complex event, multiple system failures may be present, whether through

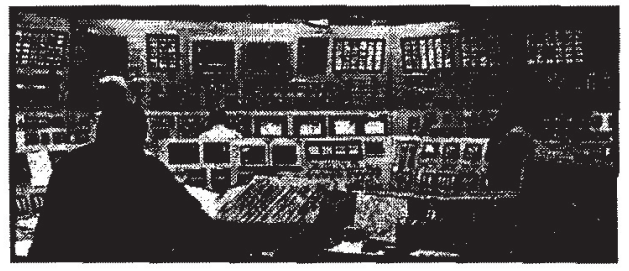

Figure 2. A nuclear power plant control room featuring both push (e.g., alarms as sen at the top of the panels) and pull (e.g., indicators, gauges, and displays as seen below the alarms) information sources for the operator. common cause or independent failures. The event complexity means the operators may experience an alarm flood, in which too many simultaneous alarms compete for the operators' attention. Not only does the number of alarms pushed to the operator saturate his or her ability to attend to individual alarms, but the ability of the operator to diagnose plant states by pulling relevant information is diminished. In the face of this flood of information, it is possible that relevant plant status indicators may be absent (e.g., through a malfunctioning sensor) or misleading (e.g., providing faulty data). While plants are designed with redundant indicators, the operator may have an overreliance on a particular indicator or may, through experience and training, look for specific indicators to confirm his or her hypotheses about what is happening at the plant.

In the context of information foraging, operators who are expecting or looking for specific information to confirm a possible but incorrect plant status may be seen as being on the wrong information scent. Control room operators, who are efficient at foraging, may ignore information that is not perceived to have a fruitful yield for their hypothesized plant state. The net result is that the operators may be seen as adopting the wrong foraging strategy in that context-a strategy that otherwise supports the activity but that yields false, incomplete, or misleading information.

\subsubsection{Display layout}

The control room operator must scan multiple indicators when pulling information. Operators can be seen as following optimal foraging strategies in searching for such information, following the shortest path with the highest information yield. Failing to provide indicators along a relevant foraging path may result in the operator consistently overlooking or ignoring those indicators, especially if those indicators are not normally meaningful and would require a search path detour under normal circumstances.

In practice, the insight provided by this observation is no different than what might be prescribed by conforming the control room to good human factors and good layout. However, the practice of designing the control room layout for indicators does not necessarily follow directly from a theoretical basis so much as from design principles, style guides, and standards derived from empirical observations. Information foraging theory provides a means to account for the optimal path of information search. Importantly, the optimal path paired with the context of operator information searches may prove predictive of the types of information that need to be incorporated into particular visual search pathways. 


\subsubsection{Automated plant functions}

Automated plant functions result in lost engagement as operators switch from a role of active controller to passive monitor of the plant system (Tran et al., 2007). In other cases, the automatic functions of the digital control system may cause the human operators to lose situation overview of the plant, potentially increasing the complexity of the operators' tasking.

Viewing plant automation from the perspective of information foraging captures these nuances. In contemporary control and monitoring of plans, operators switch between push and pull strategies. This dynamic keeps operators engaged in the plant and helps them form active hypotheses about plant states in order to maintain situation awareness Plant automation potentially degrades this switch between push and pull and forces the role of operators as spiders, receiving plant information but not necessarily shaping the outcome. The important lesson learned from this application of information foraging theory is that the dynamic roles of information search may be central to building the operators' understanding of the plant and to maintaining operator engagement.

\subsubsection{Alarm response}

Operators can quickly become overwhelmed by nuisance alarms during normal operations and alarm floods during abnormal operations (Grosdidier et al., 2003). As previously noted, each alarm triggers and requires operator response, shifting to an active pull or wolf strategy for the operators. Each alarm sends the operators on an information path, but nuisance alarms drive the operators down the wrong information path, because the alarm does not actually signify a true off-normal condition. In the case of alarm floods, the operators receive more alarms than they can respond to, and they must determine the priority of their response. (Note that in conventional annunciator displays with light boxes, the alarms do not explicitly convey their priority, although operators learn through training which alarms are highest priority.) With alarm flooding, the problem is often seen in terms of information overload. This notion is misleading, because the alarms actually push very little information. The true issue is information scent overload. The operators must pull additional information as triggered by the alarm, and the operators are confronted with too many simultaneous and competing information scents to carry out an effective foraging strategy.

Figure 3 depicts alarms for three types of plant conditions-normal, upset, and abnormal operations. Each alarm (depicted by a small circle with an outward directed arrow) implies plant move-

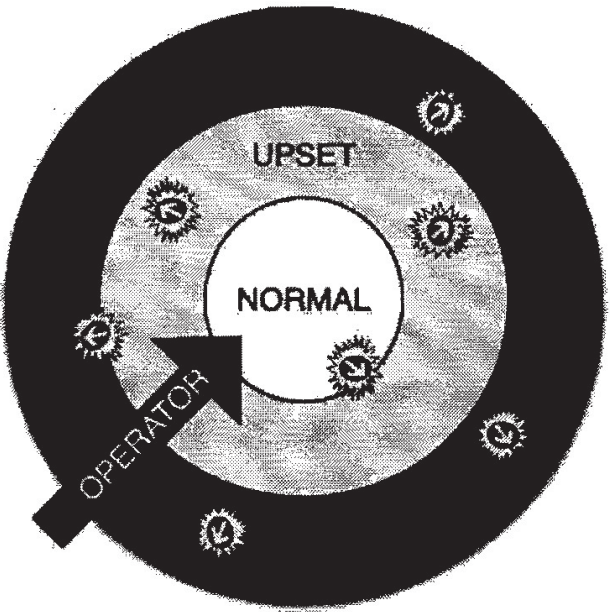

Figure 3. Alarms push information indicating a movement of the plant away from normal operations, to which the operators must respond. Each alarm represents an information scent, and the operators can become overwhelmed by having too many competing information scents.

ment toward abnormal operations, requiring the operator to respond to the upset to restore normal operations. The operator response to this alarm requires gathering (or pulling) additional information in order to make the correct diagnosis of the best response. In the case of alarms occurring during normal operations, these may be nuisance or false alarms if there is actually no plant upset. Many alarms are triggered by fixed setpoints that may not be applicable during particular modes of operation. In the case of upset or abnormal state alarms, the operators work to drive the plant state toward normal operations. With concurrent alarms, the operators cannot respond appropriately to each alarm, and they must: (i) decide which alarms to respond to first, (ii) address those aiarms, and (iii) maintain awareness of additional alarms, including processing additional alarms.

\section{IMPLICATIONS OF INFORMATION FORAGING}

\subsection{Research questions}

One of the most striking aspects of the application of information foraging to control rooms is that many well documented human factors issues can be covered by information foraging theory. The explanations are plausible but not tested in practice. Reviewing the existing research on information 
foraging as used in design and usability work in consumer software and Web interfaces (Pirolli, 2005) suggests information foraging can help optimize the display of information and the engagement of the user. These issues have not yet been tested in the context of control rooms.

To address the potential for information foraging to be applied as a useful theoretical model and practical tool for control rooms, I suggest a multistage research effort to answer the following three questions:

1. Does information foraging account for documented normal and abnormal operations in nuclear power plant control rooms? The examples provided in this paper suggest that to be the case. However, I propose a systematic mapping of operational scenarios to information foraging, including the use of information flow tied to event sequence diagrams to track how information is pushed, pulled, and acted upon by operators.

2. Does information foraging help us improve the design of control rooms? Is the theory generative of design rules, and can it help to design better systems or improve existing ones? Does information foraging tell us anything about how to present information effectively in the control room? Does informatin foraging identify performance weaknesses in our current control rooms? Much of the practice of information foraging has centered upon creating simplified information paths for searched content in Web search engines. Given the complex layout of control rooms, it is important to see if the information flow diagrams generated in the first research question can suggest new rules for effective information presentation in control rooms.

3. Is information foraging informative to human performance models? Human performance modeling is the simulation of human decisions and actions. Information foraging has already been applied to informatin foraging (e.g., SNIFACT or Scent-based Navigation and Information Foraging in the ACT architecture in Fu and Pirolli, 2007). The challenge becomes to apply this same approach to an opeator simualtion model for control rooms (e..g., Chang and Mosleh, 2007; Coyne, 2009). As the general nature of information foraging in control rooms comes to be understood through the first two research questions, these insights can be translated into a modeling framework coupled with empirical validation. This modeling framework becomes especially important for the design of new control rooms, in which human performance modeling will be used as a tool to pre-screen designs using virtual operators prior to operator-in-theloop studies and integrated system validation (Boring et al, 2008).

\section{CONCLUSIONS}

In this paper, I have provided a brief review of information foraging theory as well as examples of how the theory can account for several phenomena in nuclear power plant control room settings. I have aiso outlined three research directions that will prove fruitful for expanding the application of information foraging to control room settings. As demonstrated in this paper, information foraging already reasonably and plausibly accounts for operator actions in the control room. Beyond this preliminary, anecdotal review of the application of information foraging to control rooms, ultimately it will be important to develop research that provides new, validated insights into operator behaviors and that generates solid design guidance for improving control room design. Information foraging holds great promise in both areas.

\section{DISCLAIMER}

The opinions expressed in this paper are those of the authors and not those of the authors' sponsoring organization. This work of authorship was prepared as an account of work sponsored by an agency of the United States Government. Neither the United States Government, nor any agency thereof, nor any of their employees makes any warranty, express or implied, or assumes any legal liability or responsibility for the accuracy, completeness, or usefulness of any information, apparatus, product, or process disclosed, or represents that its use would not infringe privately-owned rights.

Idaho National Laboratory is a multi-program laboratory operated by Battelle Energy Alliance LLC, for the United States Department of Energy under Contract DE-AC07-05ID 14517.

\section{REFERENCES}

Boring, R.L., Gertman, D.I., Tran, T.Q., \& Gore, B.F. 2008. Framework for the application of MIDAS for modeling control room crew performance at nuclear power plants. Proceedings of the 52nd Annual Meeting of the Human Factors and Ergonomics Society, $930-934$.

Chang, Y.H.J., \& Mosleh, A. 2007. Cognitive modeling and dynamic probabilistic simulation of operating crew response to complex system accidents Part 1: Overview of the IDAC Model. Reliability Engineering and System Safety, 92: 997-1013. 
Coyne, K.A. 2009. A predictive model of nuclear powr plant crew decision-making and performance in a dynamic simulation environment. $\mathrm{PhD}$ Dissertation. College Park: University of Maryland.

Fu, W.-T. \& Pirolli, P. 2007, SNIF-ACT: A cognitive model of user navigation on the world wide web. Human-Computer Interaction, 22: 355-412.

Grosdidier, P., Connor, B. Hollifield, B., \& Kulkarni, S 2003. A path forward to DCS alarm management. Hydrocarbon Processing, November 2003: 59-64.

Kamil, A.C., Krebs, J.R., \& Pulliam, H.R. 1987. Foraging behavior. Plenum Press: New York.

Marlowe, F.W. 2005. Hunter-gatherers and human evolution. Evolutionary Anthropology: Issues, News, and Reviews, 14(2): 54-67.

Maslow, A.H. 1943. A theory of human motivation. Psychological Review, 50(4): 370-96.

Pirolli, P. 2005. Rational analyses of information foraging on the Web. Cognitive Science, 29, 343-373.
Pirolli, P. 2007. Information foraging theory, Adaptive interaction with information. Oxford: Oxford University Press.

Skinner, B.F. 1974. About Behaviorism, New York: Vintage.

Stephens, D.W., \& Krebs, J.R. 1986. Foraging theory. Princeton: Princeton University Press.

Tran, T.Q., Garcia, H., Boring, R.L., Joe, J.C., \& Hallbert, B.P. 2007. Human factors issues for multimodular reactor units. Official Proceedings of the Joint 8th IEEE Conference on Human Factors and Power Plants and the 13th Annual Workshop on Human Performance/Root Cause/Trending/Operating Experience/Self Assessment, 347-352.

Von Neumann, J. \& Morgenstern, O. 1947. Theory of games and economic behavior. Second edition. Princeton, NJ. Princeton University Press. 